\title{
SF3B1: hotspot mutazionale nei prolattinomi
}

\author{
Giovanna Mantovani $^{1} \cdot$ Federica Mangili $^{1}$
}

Accettato: 25 gennaio 2021 / Pubblicato online: 14 maggio 2021

(c) The Author(s) 2021

\section{Commento a:}

\section{Somatic SF3B1 hotspot mutation in prolactinomas.} C. Li, W. Xie, J.S. Rosenblum, J. Zhou, J. Guo, Y. Miao, Y. Shen, H. Wang, L. Gong, M. Li, S. Zhao, S. Cheng, H. Zhu, T. Jiang, S. Ling, F. Wang, H. Zhang, M. Zhang, Y. Qu, Q. Zhang, G. Li, J. Wang, J. Ma, Z. Zhuang, Y. Zhang. Nature Communications (2020) 11:2506

In termini di prevalenza, i prolattinomi rappresentano all'incirca la metà dei tumori ipofisari e sono caratterizzati dall'ipersecrezione di prolattina (PRL) correlata a sintomi endocrinologici. La terapia di prima linea prevede l'impiego di agonisti della dopamina; tuttavia, una parte di essi risulta esserne resistente e il meccanismo alla base di questa natura aggressiva non è stato ancora del tutto definito [1].

Ad oggi, i possibili eventi genetici determinanti i tumori ipofisari risultano poco chiari e solamente alcune mutazioni sono state identificate, tra cui quelle dei geni GNAS, USP8, USP48 e BRAF [2, 3]. Nello specifico, non sono riportate alterazioni genetiche ricorrenti nei prolattinomi, fattore che ha limitato ulteriormente la comprensione della patogenesi e, di conseguenza, l'eventuale sviluppo di altre strategie terapeutiche mirate.

Il lavoro oggetto del presente Commento ha previsto uno studio genomico su campioni di prolattinomi umani rimossi chirurgicamente con il fine di identificare nuovi geni associati allo sviluppo di questi tumori.

Come primo punto è stata condotta un'analisi di sequenziamento genomico (Whole Genome Sequencing) su una pri-

G. Mantovani

giovanna.mantovani@unimi.it

1 Unità di Endocrinologia, Fondazione IRCCS Ca' Granda Ospedale Maggiore Policlinico; Dipartimento di Scienze Cliniche e di Comunità, Università degli Studi di Milano, Milano, Italia ma serie di 21 tumori che ha rivelato una mutazione nel gene $\mathrm{SF} 3 \mathrm{~B} 1^{\mathrm{R} 625 \mathrm{H}}$ in due casi. Successivamente, tale mutazione è stata confermata tramite digital PCR e l'ampliamento della casistica ha portato a un suo riscontro in 45 di 227 campioni analizzati, corrispondente a una frequenza del 19,8\%. Inoltre, importante sottolineare che la mutazione non è stata evidenziata in altre tipologie di tumori ipofisari (tireotropi, somatotropi, gonadotropi, corticotropi e null cell), eccezion fatta per due casi misti, risultati però immuno-positivi per PRL. Alla luce di queste analisi, la mutazione di SF3B1 ${ }^{\mathrm{R} 625 \mathrm{H}}$ appare una peculiare firma genetica dei tumori PRL-secernenti.

Filogeneticamente, il locus $\mathrm{SF} 3 \mathrm{~B} 1^{\mathrm{R} 625 \mathrm{H}}$ risulta essere molto conservato tra le specie, suggerendone un ruolo essenziale nell'espressione genica e nella regolazione posttrascrizionale.

Per determinare il ruolo della mutazione nei prolattinomi sono stati testati i livelli di secrezione, proliferazione e apoptosi cellulare in culture primarie e su linee cellulari murine PRL-secernenti. I dati hanno evidenziato un significativo aumento di PRL e di proliferazione cellulare, e una riduzione di apoptosi in tumori SF3B $1^{\mathrm{R} 625 \mathrm{H}}$ mutati. Attraverso ulteriori esperimenti è emerso come la mutazione $\mathrm{SF} 3 \mathrm{~B} 1^{\mathrm{R} 625 \mathrm{H}}$ tramite splicing alternativo risulti in un'acquisizione di funzione di estrogen-related receptor gamma (ESRRG), le cui funzioni biologiche sono strettamente coinvolte nelle vie di segnale estrogenico, notoriamente coinvolte nella sintesi di PRL e nella proliferazione di cellule lattotrope. La mutazione SF3B $1^{\mathrm{R} 625 \mathrm{H}}$ promuove dunque un'ipersecrezione di PRL attraverso uno splicing aberrante di ESRRG.

In ultimo, sono state analizzate le caratteristiche cliniche dei 227 pazienti esaminati, evidenziando un'associazione tra la mutazione di SF3B $1^{\mathrm{R} 625 \mathrm{H}}$ a una maggiore secrezione di PRL (PRL plasmatica/volume tumorale) e una riduzione della sopravvivenza libera da progressione rispetto 
al gruppo wild-type. In due casi è stata inoltre evidenziata la presenza di un comportamento clinicamente maligno.

In conclusione, questo studio ha permesso l'identificazione di SF3B1 ${ }^{\mathrm{R} 625 \mathrm{H}}$ come nuovo hotspot mutazionale causativo di un sottogruppo di tumori PRL-secernenti. Il presente studio elucida altresì un potenziale meccanismo cellulare alla base della patogenesi dei prolattinomi che coinvolge le vie di traduzione del segnale mediate dagli estrogeni e che potrebbe condurre allo sviluppo di nuove terapie farmacologiche mirate.

Funding Note Open access funding provided by Università degli Studi di Milano within the CRUI-CARE Agreement.

Nota della casa editrice Springer Nature rimane neutrale in riguardo alle rivendicazioni giurisdizionali nelle mappe pubblicate e nelle affiliazioni istituzionali.

Open Access This article is licensed under a Creative Commons Attribution 4.0 International License, which permits use, sharing, adaptation, distribution and reproduction in any medium or format, as long as you give appropriate credit to the original author(s) and the source, provide a link to the Creative Commons licence, and indicate if changes were made. The images or other third party material in this article are included in the article's Creative Commons licence, unless indicated otherwise in a credit line to the material. If material is not included in the article's Creative Commons licence and your intended use is not permitted by statutory regulation or exceeds the permitted use, you will need to obtain permission directly from the copyright holder. To view a copy of this licence, visit http://creativecommons.org/licenses/by/4.0/.

\section{Bibliografia}

1. Molitch ME (2017) Diagnosis and treatment of pituitary adenomas: a review. JAMA 317:516-524

2. Landis CA, Masters SB, Spada A et al (1989) GTPase inhibiting mutations activate the alpha chain of Gs and stimulate adenylyl cyclase in human pituitary tumours. Nature 340:692-696

3. Chen J, Jian X, Deng S et al (2018) Identification of recurrent USP48 and BRAF mutations in Cushing's disease. Nat Commun 9:3171 